\title{
Exploring the non-linear motion of the parsec-scale jet of FSRQ 1633+382
}

\author{
Hyunwook Ro* \\ Department of Astronomy, Yonsei University, Korea \\ Korea Astronomy and Space Science Institute, Korea \\ E-mail: hwro@yonsei.ac.kr
}

\section{Bong Won Sohn}

Korea Astronomy and Space Science Institute, Korea

E-mail: bwsohn@kasi.re.kr

\section{Aeree Chung}

Department of Astronomy, Yonsei University, Korea

E-mail: achungeyonsei.ac.kr

\section{Thomas P. Krichbaum}

Max Plank Institute für Radioastronomie, Germany

E-mail: tkrichbaum@mpifr-bonn.mpg • de

\begin{abstract}
Helical magnetic field threading the accretion disk and the black hole likely play a key role in jet launching mechanisms in active galactic nuclei (AGNs). The flat-spectrum radio quasar (FSRQ) $1633+382$ is a radio-loud AGN with well-developed jets which have been monitored with very long baseline interferometry (VLBI) over the last few decades, making an excellent target to probe the structure of magnetic fields in the parsec (pc)-scale quasar jets. From its VLBI observations over two decades, we find evidence for quasi-periodic and oscillating patterns in the positions of some jet components and their luminosities. The patterns are well explained when the jet components are assumed to be moving along helical trajectories, changing the Doppler factor and the apparent speed as the viewing angle of the jet changes. Our analysis supports the presence of a helically ordered magnetic field along the jet, which is predicted by jet theories and also suggested by previous observations.
\end{abstract}

14th European VLBI Network Symposium \& Users Meeting (EVN 2018)

8-11 October 2018

Granada, Spain

${ }^{*}$ Speaker. 


\section{Introduction}

Currently, in the most favored paradigm of AGN jet formation, jets are powered by the rotational energy released by the accretion disk and/or super-massive black hole. In addition, strong, large-scale helically ordered magnetic fields seem to be required in the jet launching, acceleration and collimation process as shown by numerical simulations (see Tcheckovskoy 2015 and references therein). Therefore, most of jetted AGNs are believed to be accompanied by helical magnetic field.

To date, many observational attempts have been made using very long baseline interferometers (VLBI) to characterize the detailed structures of the magnetic field near the black hole (see Gabuzda 2015 and references therein). One way is to observe radio polarization angle swinging as jet component propagates downward (e.g. Marscher et al. 2008; Britzen et al. 2017; Myserlis et al. 2018). Another way is to observe the change of the Faraday rotation measure across jets (e.g. Hovatta et al. 2012; Zamaninasab et al. 2013; Gabuzda et al. 2014). Alternatively, the kinematics of jets can be used to compare the motion of jet components with that predicted by helical trajectory models (e.g. Steffen 1995; Liu et al. 2010; Molina et al. 2014). However, many previous observations still suffered from the limited angular resolution and the de-polarization due to ambient medium, and the direct observational evidence for the helical magnetic field in the VLBI scale jet is still rare (see e.g. Taylor and Zavala 2010).

FSRQ $1633+382$ is a flat spectrum radio quasar at $\mathrm{z}=1.814$. It has been observed in a range of radio frequencies using the VLBI over a long period of time (e.g. Lister et al., 2016; Jorstad et al. 2017), providing an ideal target for examining the characteristics of pc-scale AGN jets. Its magnetic field in the jet base is sufficiently strong to make a dynamical impact on the accretion flow (1.62 Gauss; Zamaninasab et al. 2014). A transverse gradient of rotation measure has been observed across the jet, hinting the presence of the helically ordered magnetic jet (Gabuzda et al. 2014).

For this target, non-linear motions of the jet components were also reported in VLBI observations at various frequencies (e.g. Liu et al. 2010; Lister et al. 2013; Jorstad et al. 2017). Particularly, Liu et al. (2010) attempted to explain those non-linear motions using a helical jet model. Due to a short time baseline however, the helical movement of the jet components was not fully conclusive. Therefore in this work, we challenge to better confirm the presence of a helical jet in FSRQ $1633+382$ using observations over a four times longer period than previously probed.

\section{Jet kinematics of FSRQ $1633+382$ over 24 years}

In order to investigate long-term structural changes in the jet of FSRQ $1633+382$, we use 81 sets of Very Long Baseline Interferometry (VLBI) archival data observed from 1994 to 2018 at 15 and $22 \mathrm{GHz}$. Among them, 14 epochs at 22GHz from 2002 June 12 to 2005 February 03 were conducted with a combined array between VLBA and Effelsberg 100m antenna in Germany (PI: T. P. Krichbaum). The initial amplitude and phase calibrations were done on the $u v$ data using Astronomical Image Processing System (AIPS). Then the data were imaged using the Difmap package. For all 53 epochs at $15 \mathrm{GHz}$, the self-calibrated visibility data were available from the MOJAVE project, which we utilize for this work (e.g. Lister et al. 2013). 
To determine the position and the flux of individual jet components, we fitted multiple 2dimensional Gaussian components to the self-calibrated visibility data using the modelfit program, which is implemented in the Difmap package. Errors of the position and the flux of Gaussian components were estimated following Lister et al. (2013), i.e. one fifth of the synthesized beam and $5 \%$ of the peak flux, respectively. For 14 epochs before 2002 at $22 \mathrm{GHz}$, we adopt the published Gaussian model fitting results (Liu et al. 2010; Jorstad et al. 2001).

In this work, the core-shift between 15 and $22 \mathrm{GHz}$ (i.e., the offset among different frequencies due to distinct synchrotron absorption) was not corrected as it is small compared to the positional error of jet components. To test this, we inspected the two-dimensional cross-correlation of two closely observed datasets in 15 and $22 \mathrm{GHz}$ (Croke and Gabuzda 2008). As a result, we find that the offset between two frequencies is 10 to 100 times smaller than a typical position error of jet components, and therefore the core-shift can be ignored.

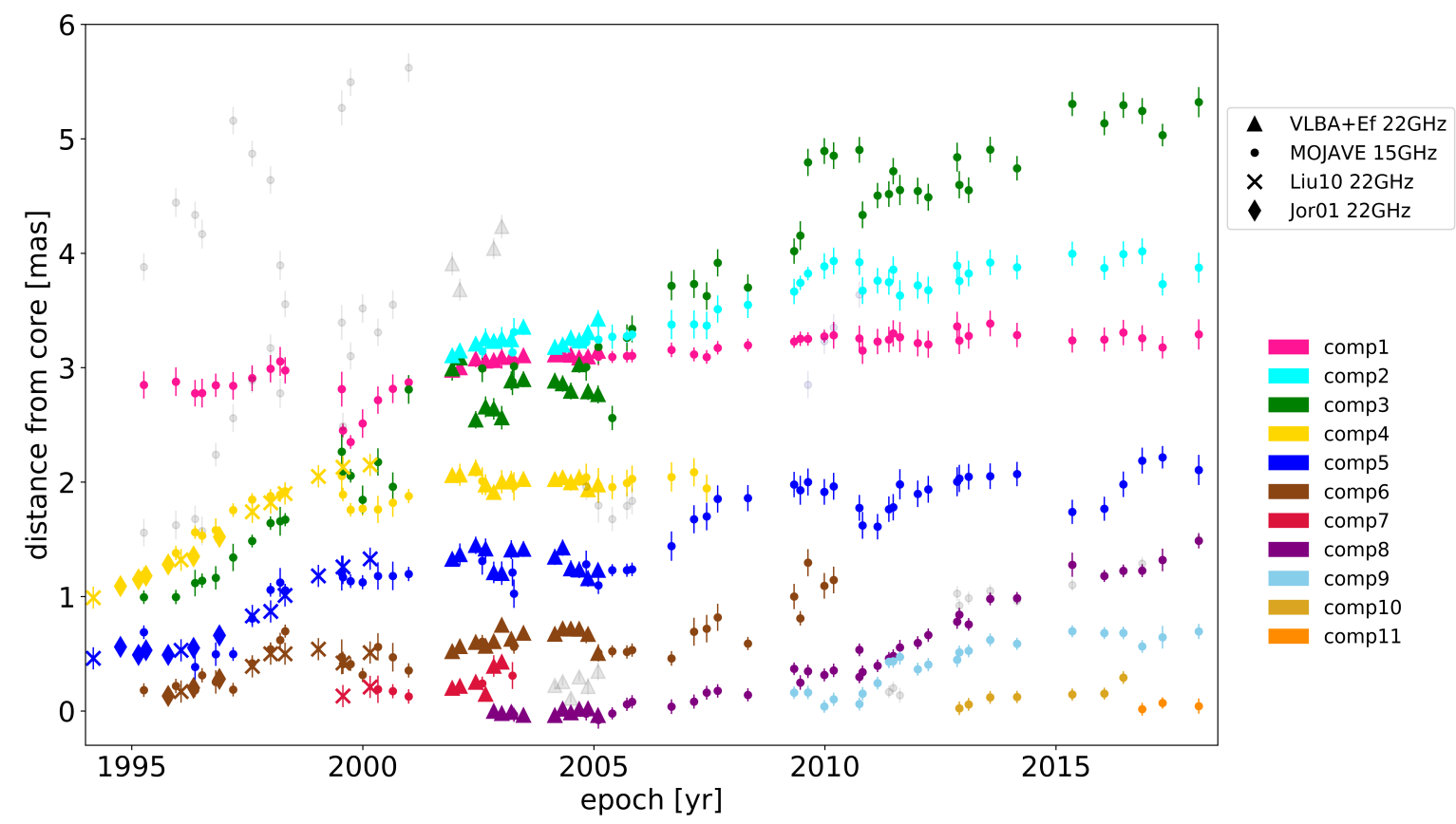

Figure 1: Radial separation of the jet components from the VLBI core of FSRQ 1633+382 versus time. Data are colored by component. Grey components are not identified as jet components. Triangles: 22GHz VLBA and Effelsberg joint array (PI: T. P. Krichbaum). Circles : 15GHz MOJAVE project. Crosses and diamonds : 22GHz published Gaussian model fitting results (Liu et al. 2010; Jorstad et al. 2001)

\section{Helical motions of jet components}

Figure 1 shows the radial distance from the core at different epochs of 11 components along FSRQ 1633+382's jet identified by the fitting. Different colors represent individual jet components. The VLBI jet can be traced up to separation of $\sim 5$ milli-arcsecond (mas) or $\sim 43 \mathrm{pc}$ in projection. For a typical viewing angle of FSRQ ( $\sim 1.5$ degrees; Jorstad et al. 2017), the de-projected jet length 
is $\sim 1.6 \mathrm{kpc}$. Intriguingly, most of the components, especially the ones within 2 mas from the core, show systematic oscillation around a well defined slope in the radial distance plot as shown in Figure 2. This is highly suggestive of non-ballistic motion.

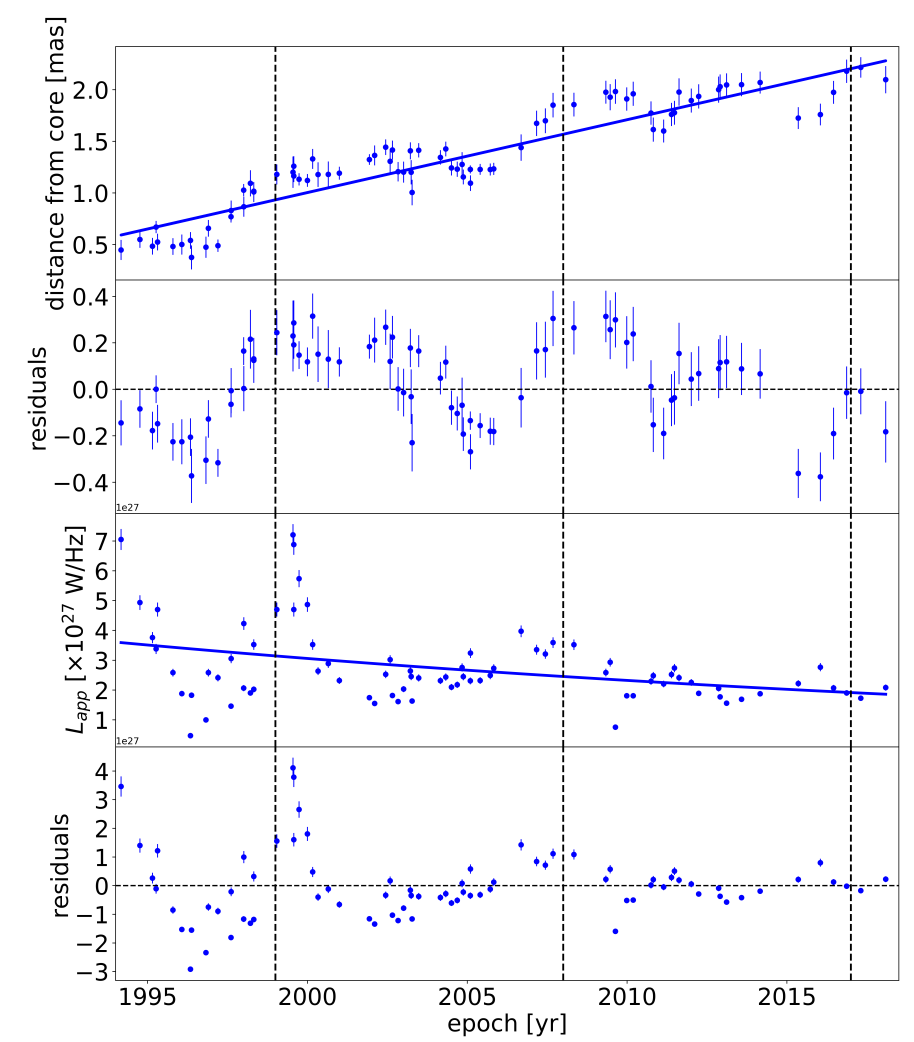

Figure 2: The radial distance (top row) and the luminosity (3rd row) variations of Component 5 (blue in Figure 1). Solid lines are the best fit which represents the general trend of increasing distance and decreasing luminosity. Residuals of the radial distance and the luminosity represent in the 2nd and 4th rows from the top, respectively. The residual shows a quasi-periodic behaviors in both distance and luminosity in a similar way. Vertical dashed lines show a 9 years of separation, which roughly corresponds to the quasi-period trend.

In order to better probe the motion of each component, both the position and the luminosity should be considered. Figure 2 shows the time variation of the position and the luminosity of Component 5 (blue in Figure 1). As the position of the emanating component moves outward with time, the luminosity decreases likely due to the radiative and/or adiabatic energy loss. The solid lines indicate the best fit which overall shows how the luminosity decreases as the component moves outward linearly. Meanwhile, an oscillatory motion of the component can be seen in the residual. The luminosity is observed to rise and decline correspondingly, as also seen in the residual.Intriguingly, both residuals seem to have a quasi-periodic trend with a maximum period of 9 years.

These periodic trends can be explained by a jet component spiraling at a constant speed with a fixed pitch angle, and a constant offset between the rotational axis and the observer's line of sight (Figure 3). When the component approaches toward us, both the luminosity and the speed 

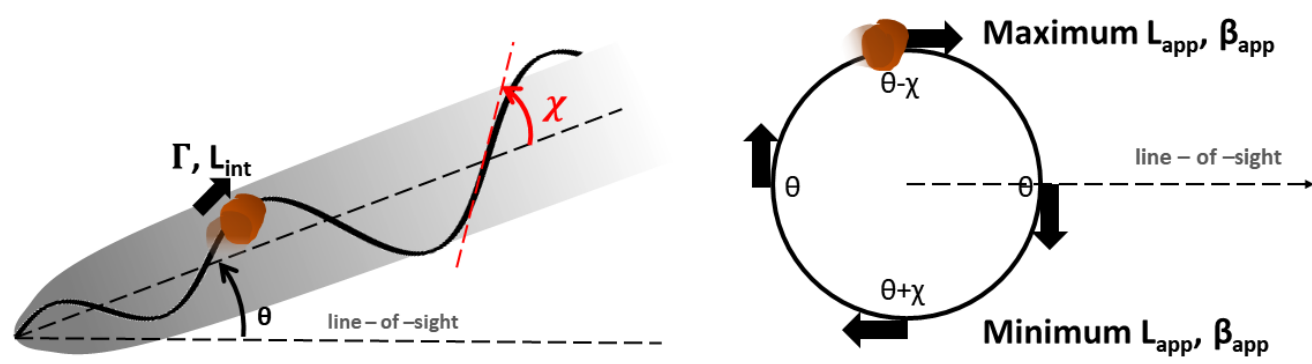

Figure 3: Schematic picture of a helical trajectory model: a) an edge-on view and b) a face-on view. A component with a constant Lorentz factor $\Gamma$ follows a helical trajectory which represents as a pitch angle of $\chi$, and the axis of the helix off from observer's line of sight with a constant angle of $\theta$. As the component moves along the helical trajectory, the angle between the component's velocity and the line-of-sight changes from $\theta-\chi$ to $\theta+\chi$, making a periodic behavior in the observed luminosity and the apparent speed, simultaneously.

will be Doppler boosted, and vice versa. Since helical motion should be strongly related to the helical magnetic field in the jet, this kinematical information allows us to probe the magnetic field structure along the AGN jet. To do so, we will quantify the observed luminosity and distance of jet components, then compare with a model where they move along a helical orbit with a fixed pitch and viewing angles with respect to the observer. In addition, we will probe the polarization structure of the jet, which is also a good way to confirm the presence of a helical magnetic field.

On the other hand, the observed quasi-periodic behavior of jet components can be also explained by a completely different mechanism such as "jet wobbling" (i.e. changes in the component ejection direction; see Agudo 2009). The wobbling motion of the jet may result from precession the accretion disk, orbital motion of the accretion system (e.g. a binary black hole system), or jet instabilities (e.g. Kelvin-Helmholtz or current-driven instabilities). In such a wobbling jet, the relative position of the jet component from the jet base also changes, and consequently the periodicity of the jet component can be observed. However, it is difficult to explain the periodic change of luminosity of the jet's emanating component only by the wobbling of the jet base. Alternatively, jet components may be moving along helical trajectories, which are precessing as shown by a recent studies of FSRQ 1308+326 (Britzen et al. 2017). Therefore, we cannot completely rule out the possibility of wobbling of $1633+382$ 's jet, which needs to be further tested.

\section{References}

[1] A. Tchekhovskoy, 2015, in Astrophys. Space Sci. Libr., 414, The Formation and Disruption of Black Hole Jets, ed. I. Contopoulos et al., Springer International Publishing, Switzerland, 45

[2] D. C. Gabuzda. 2015, in Astrophys. Space Sci. Libr., 414, The Formation and Disruption of Black Hole Jets, ed. I. Contopoulos et al., Springer International Publishing, Switzerland, 117

[3] A. P. Marscher, 2008, Nature, 452, 966

[4] S. Britzen, et al., 2017, A\&A, 602, 29 
[5] I. Myserlis, et al., 2018, $A \& A, \mathbf{6 1 9}, 88$

[6] T. Hovatta, et al., 2012, AJ, 144, 105

[7] M. Zamaninasab, et al., 2013, MNRAS, 436, 3341

[8] D. C. Gabuzda, A. R. Reichstein, E. L. O’Neill, 2014, MNRAS, 444, 172

[9] W. Steffen, et al., 1995, A\&A, 302, 335

[10] Y. Liu, D. R. Jiang, Z. Q. Shen, M. Karouzos, 2010, A\&A, 522, 5

[11] S. N. Molina, et al., 2014, A\&A, 566, 26

[12] G. B. Taylor, R. Zavala, 2010, ApJ, 722, 183

[13] M. L. Lister, et al., 2016, AJ, 152, 12

[14] S. G. Jorstad, et al., 2017, ApJ, 846, 98

[15] M. Zamaninasab, E. Clausen-Brown, T. Savolainen, A. Tchekhovskoy, 2014, Nature, 510, 126

[16] M. L. Lister, et al., 2013, AJ, 146, 120

[17] S. G. Jorstad, et al., 2001, ApJS, 134, 181

[18] S. M. Croke, D. C. Gabuzda, 2008, MNRAS, 386, 619

[19] I. Agudo. 2009, in ASP Conf. Ser, 402, Approaching Micro-Arcsecond Resoluion with VSOP-2: Astrophysics and Technology, ed. Y. Hagiwara et al., 330 